\title{
Bandgap analysis of two-dimensional phononic crystals
}

\author{
Feng-lian $\mathrm{Li}^{1, \mathrm{a}}$ \\ ${ }^{1}$ School of Electromechanical engineering, Beijing Information Science and Technology \\ University, Beijing, China \\ aemail: lifenglian@126.com
}

\begin{abstract}
Keywords: Phononic crystals; Band gaps; Interface conditions
Abstract. The Dirichlet-to-Neumann method is adopted to calculate the band gaps of the two-dimensional phononic crystals with different interface conditions. The system is in a square lattice which is composed of the solid cylinders embedded in a softer material. The cross sections of the inclusions are circular. In a unit cell a linear eigenvalue equation is formulated, from which the band gaps can be obtained. A typicla numerical example is taken to analyze and discuss the roles of the interface conditions. The results show that the Dirichlet-to-Neumann method can provide accurate results and the different interface conditions have significant effects on the band gaps.
\end{abstract}

\section{Introduction}

In the last years, a great deal of work has been devoted to the study of photonic crystals, which consists of periodic arrays with dielectric materials on small scale of light wavelength. They can control the electromagnetic waves because of the existence of band gaps [1-3]. Analogously, the phononic crystal is an artificial crystal omposed of two or more elastic materials with different mass densities and elastic properties. The research emphasis was the existence of complete or directional elastic band gaps, over which there are no elastic waves propagating through the lattice. The complete or directional band gaps are helpful in the design of new generations of sound shields, transducers, filters, refractive devices such as acoustic interferometers and acoustic lenses, etc. Furthermore, phononic crystals are also useful in the design of vibrationless environments for high-precision mechanical systems [4-6]. Therefore, The study of phononic crystals has become one of the most fast-developing and active fields in acoustics, condensed matter physics, mechanical engineering, mechanics, etc [7,8].

So far, many methods have been used to compute the band structures of elastic/acoustic waves in phononic crystals. For example, the plane-wave expansion method [9] is widely used to compute the band gaps of the phononic crystals, but it has the slow convergence when it deals with the systems of very high and very low filling ratios, and cannot consider the interface conditions of the matrix and the inclusions. The finite difference time domain method [10] and the finite element method [11] have good convergence in the calculations of phononic crystals, but their formed matrix is large and time-consuming. The multiple-scattering theory method [12] has a larger multipole expansion, and it can only treat the cylindrical or spherical inclusions. The wavelet method [13] need adopt suitable basis functions and consume more time.

The recently developed Dirichlet-to-Neumann (DtN) map method [14-16] has been used to calculate the band gaps of the two-dimensional phononic crystals. This method is based on the cylindrical wave expansion, which is an operator that maps the wave field such as the displacement to its normal derivative on the boundary of a unit cell. It yields a linear eigenvalue problem, where the Bloch wave vector is an eigenvalue. In comparisons with the other methods, the DtN method has small matrices and good convergence, and it can treat the different interface conditions between the matrix and the inclusion. In this paper we will use the DtN method to compute the band gaps of two-dimensional phononic crystals with soft components under the different interface conditions, and analysis the effects of the interface conditions on the band gaps. 


\section{Wave equations and method}

The phononic crystal, which is composed solid cylinders embedded in the solid matrix forming a square lattice with the lattice constant $a$, is shown in Fig. 1 where the corresponding unit cells and the first Brillouin zones are given. The cross-sections of the inclusions are circular. We suppose that the propagation of the elastic waves is limited to the transverse plane. As we know, the governing equations describing these time-harmonic wave motions can be expressed as

$$
\mu_{j} \nabla \cdot\left(\nabla \mathbf{u}_{j}(\mathbf{x})\right)+\mu_{j} \nabla \cdot\left(\frac{\partial}{\partial x_{i}} \mathbf{u}_{j}(\mathbf{x})\right)+\lambda_{j} \frac{\partial}{\partial x_{i}}\left[\nabla \cdot \mathbf{u}_{j}(\mathbf{x})\right]+\rho_{j} \omega^{2} \mathbf{u}_{j}(\mathbf{x})=0, \quad \mathbf{x} \in D_{j}
$$

where $\mathbf{x}=(x, y) ; \mathbf{u}_{j}(\mathbf{x})=\left(u_{j x}, u_{j y}\right) ; \nabla=(\partial / \partial x, \partial / \partial y) ; \omega$ is the angular frequency; $\lambda_{j}, \mu_{j}$ and $\rho_{j}$ are the Lamé coefficients and mass density of the inclusions and the matrix, respectively; $j=1$ for the solid inclusions ; and $j=0$ for the solid matrix.

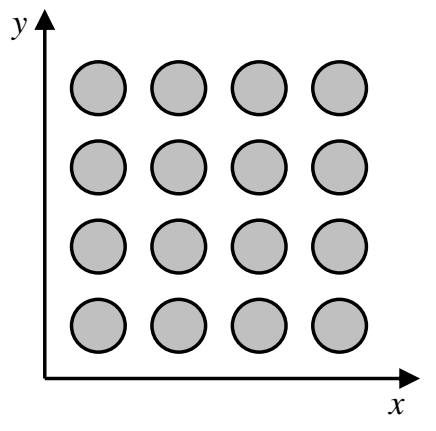

(a)

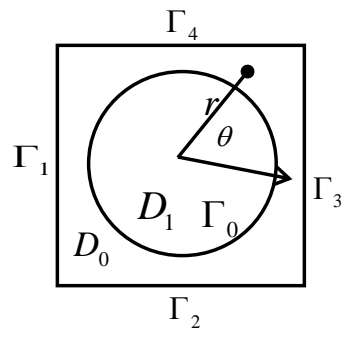

(b)

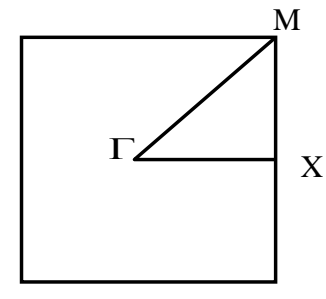

(c)

Fig.1 (a) is a square lattice; (b) is its corresponding square unit cell and (c) is the first Brillouin zone.

In a unit cell (Fig. 1(b)), when the matrix and the inclusion smoothly contact, the interface conditions can be expressed as the following relations

$$
\left\{\begin{array}{l}
u_{r 1}(r, \theta)=u_{r 0}(r, \theta), \\
\sigma_{r \theta 1}(r, \theta)=\sigma_{r \theta 0}(r, \theta), \quad r=R . \\
\sigma_{r r 1}(r, \theta)=\sigma_{r r 0}(r, \theta), \\
\sigma_{r \theta 0}(r, \theta)=0
\end{array}\right.
$$

When the matrix and the inclusion perfectly contact, we have the following interface conditions

$$
\left\{\begin{array}{l}
u_{\theta 1}(r, \theta)=u_{\theta 0}(r, \theta), \\
u_{r 1}(r, \theta)=u_{r 0}(r, \theta), \quad r=R . \\
\sigma_{r \theta 1}(r, \theta)=\sigma_{r \theta 0}(r, \theta), \\
\sigma_{r r 1}(r, \theta)=\sigma_{r r 0}(r, \theta),
\end{array}\right.
$$

According to the Bloch theorem, any field quantity $p$ (e.g. the displacements and stresses) have the following relations

$$
p(x, y)=e^{\mathbf{k} \cdot \mathbf{x}} p(x, y),
$$

in which $\mathbf{k}$ is a Bloch wave vector; $i=\sqrt{-1} . p(x, y)$ is a periodic function.

Here, we adopt the DtN method to compute the band gaps of the aboved problems. The descriptions of the method can be found in Ref. [15]. 


\section{Numerical examples}

Now, we consider a square lattice of Au cylinders embedded in the rubber matrix. The density and the wave velocities are: $\rho_{1}=19500 \mathrm{~kg} / \mathrm{m}^{3}, c_{l, 1}=3360 \mathrm{~m} / \mathrm{s}$ and $c_{t, 1}=1239 \mathrm{~m} / \mathrm{s}$ for $\mathrm{Au}$; and $\rho_{0}=1000 \mathrm{~kg} / \mathrm{m}^{3}$, $c_{l, 0}=1830 \mathrm{~m} / \mathrm{s}$ and $c_{t, 0}=500 \mathrm{~m} / \mathrm{s}$ for the rubber. The lattice constant is $a=1.0 \mathrm{~mm}$ and the filling fraction of the rubber is $f=0.21$. In the computation, we choose 8 discretized nodes on each side of the matrix which can give convergent results. The band structures with the smooth interface condition (2) are plotted in Fig. 2 by the solid scattered dots. At the same time, the band structures with the perfect interface condition (3) are shown in the figure by the hollow scattered dots. From Fig.2 we can find that except the third band all the bands for the smooth interface are lower than those for the perfect interface at the given frequency interval. Because the third band rises, a complete band gap [0.411, 0.617] exists between the second and third bands for the smooth interface, which exists between the third and fourth bands for the perfect interface. In contrast with the band gaps obtained by the two interface conditions, it is shown that the complete band gaps for the smooth interface (2) is lower and narrower than that for the perfect interface (3). Based on the material parameters, as the rubber is a softer material; the shear stress is not easy to be transmitted, especially when the interface is smooth. This example proves that the DtN map method can compute the band structures of the two-dimensional phononic crystals with softer components under the different interface conditions and give accurate results.

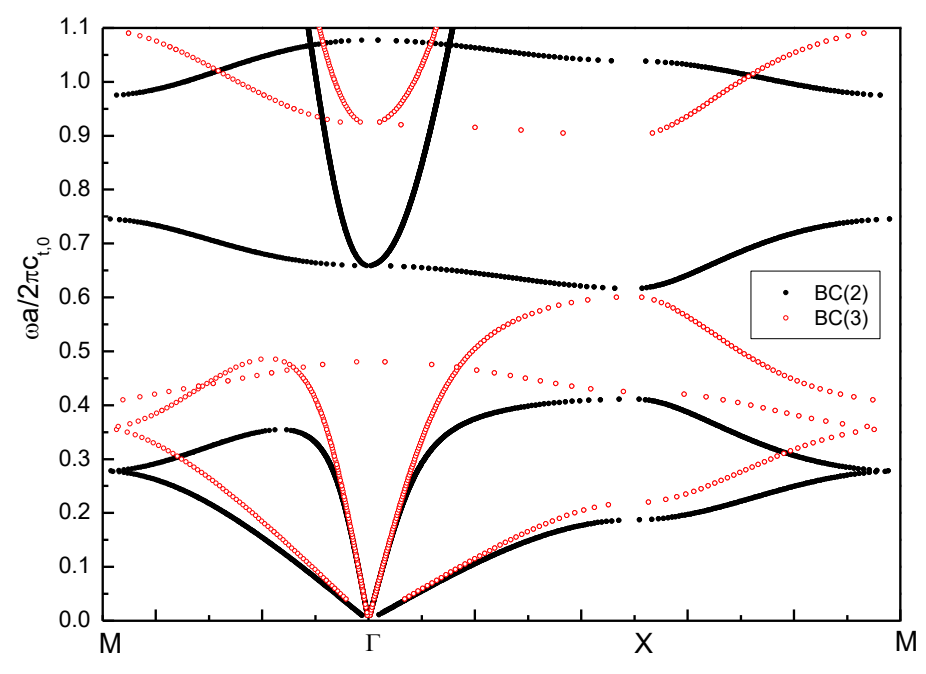

Fig. 2 Band structures of the Au/rubber phononic crystal in a square lattice. The solid and hollow scattered dots represent the results of the smooth interface condition and the perfect interface condition, respectively.

\section{Conclusions}

The Dirichlet-to-Neumann map method is applied to calculate band structures of the two-dimensional phononic crystal with the smooth and perfect interface conditions. The system is composed of $\mathrm{Au}$ and rubber materials, in which rubber is a softer material. By the computation on the band structures of the system, the perfect interface opens a complete band gap between the third and fourth bands, while the smooth interface opens a complete band gap between the second and third bands. Additionally, the band gap for the smooth interface is lower and narrower. Therefore, the interface conditions have significant effects on the band gaps. 


\section{Acknowledgements}

This work was financially supported by the National Natural Science Foundation of China (Grant No. 11202021), the Beijing Natural Science Foundation (No. 1163008), and the Postdoctoral Science Foundation of China (2012M510311).

\section{References}

[1] P. Sheng: Scattering and Localization of Classical Waves in Random Media (World Scientific, Singapore, 1990).

[2] J.D. Joannopoulos, R.D. Meade and J.N. Winn: Photonic Crystals: Molding the Flow of Light (Princeton University Press, Princeton, 1995 )

[3] E. Yablonovitch: Phys. Rev. Lett. 58 (1987), P. 2059.

[4] M.S. Kushwaha, P. Halevi and G. Martinez: Phys. Rev. Lett. 71 (1993), P. 2022.

[5] Z. Liu, C.T. Chan and P. Cheng: Phys. Rev. B 65 (2002), p. 165116.

[6] C.J. Rupp, A. Evgrafov, K. Maute and M.L. Dunn: Struct. Multi. Opt. 34 (2007), P.111.

[7] C. Q. Chen, J. Z. Cui, H. L. Duan, X. Q. Feng, L. H. He, G. K. Hu, M. J. Huang, Y. Z. Huo, B. H. Ji, B. Liu, X. H. Peng, H. J. Shi, Q. P. Sun, J. X. Wang, Y. S. Wang, H. P. Zhao, Y. P. Zhao, Q. S. Zheng and W. N. Zou: Acta Mech. Solida Sinica 24 (2011), P. 1.

[8] J.O. Vasseur, P.A. Deymier and M. Beaugeois: Zeitschrift für Kristallographie 220 (2005), P. 829.

[9] Y. J. Cao, Z. L. Hou and Y.Y. Liu: Phys. Lett. A 327 (2004), P. 247.

[10] Y.J. Cao, Z.L. Hou and Y.Y. Liu: Solid State Commun. 132 (2004), P. 539.

[11] Y.F. Wang, Y.S. Wang and X.X. Su: J. Appl. Phys. 110 (2011), P. 113520.

[12]F. C. Hsu, T.T. Wu, J.C. Hsu and J.H. Sun: Appl. Phys. Lett. 93 (2008), P. 201904.

[13]Z.Z. Yan, Y.S. Wang and Ch. Zhang: Acta Mech. Solida Sin. 21 (2008), P. 104.

[14]F.L. Li, Y.S. Wang and Ch. Zhang: Phys. Scrip 84 (2011), p. 055402.

[15] N. Zhen, F.L. Li, Y.S. Wang and Ch. Zhang: Acta Mech. Sinica 28 (2012), p.1143.

[16] N. Zhen, Y.S. Wang and Ch. Zhang: Phys. E 54 (2013), p. 125. 